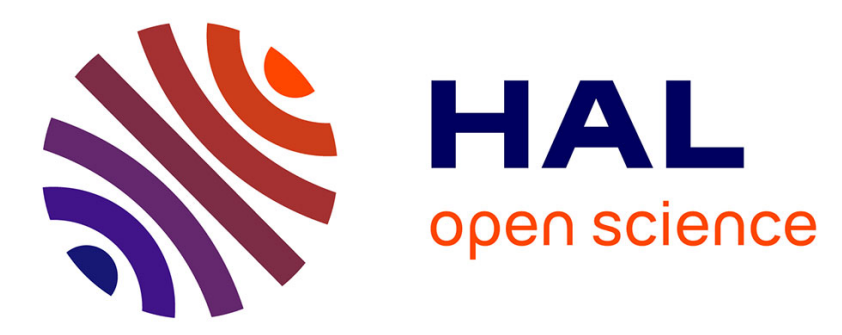

\title{
Mode dependent vector quantization with a rate-distortion optimized codebook for residue coding in video compression
}

Bihong Huang, Felix Henry, Christine Guillemot, Philippe Salembier

\section{- To cite this version:}

Bihong Huang, Felix Henry, Christine Guillemot, Philippe Salembier. Mode dependent vector quantization with a rate-distortion optimized codebook for residue coding in video compression. IEEE Intl. Conf. on Acoustics and Signal Processing (IEEE-ICASSP), Apr 2015, Brisbane, Australia. hal-01204768

\section{HAL Id: hal-01204768 \\ https://hal.science/hal-01204768}

Submitted on 24 Sep 2015

HAL is a multi-disciplinary open access archive for the deposit and dissemination of scientific research documents, whether they are published or not. The documents may come from teaching and research institutions in France or abroad, or from public or private research centers.
L'archive ouverte pluridisciplinaire HAL, est destinée au dépôt et à la diffusion de documents scientifiques de niveau recherche, publiés ou non, émanant des établissements d'enseignement et de recherche français ou étrangers, des laboratoires publics ou privés. 


\title{
MODE DEPENDENT VECTOR QUANTIZATION WITH A RATE-DISTORTION OPTIMIZED CODEBOOK FOR RESIDUE CODING IN VIDEO COMPRESSION
}

\author{
Bihong Huang $^{1,2}$, Felix Henry ${ }^{1}$, Christine Guillemot $^{2}$, Philippe Salembier $^{3}$ \\ ${ }^{1}$ Orange Labs \\ 4 Rue du Clos Courtel, 35512 Cesson Sevigne, France \\ ${ }^{2}$ INRIA \\ Campus de Beaulieu, 35042 Rennes Cedex, France \\ ${ }^{3}$ Universitat Politecnica de Catalunya \\ Jordi Girona 1-3, 08034 Barcelona, Spain
}

\begin{abstract}
The High Efficiency Video Coding standard (HEVC) supports a total of 35 intra prediction modes which aim at reducing spatial redundancy by exploiting pixel correlation within a local neighborhood. In this paper, we show that spatial correlation remains after intra prediction, leading to high energy prediction residues. We propose a novel scheme for encoding the prediction residues using a Mode Dependent Vector Quantization (MDVQ) which aims at reducing the redundancy in residual signals. The MDVQ codebook is optimized in a rate-distortion (RD) sense. Experimental results show that the codebook can be independent of the quantization parameter (QP) with no loss in terms of coding efficiency. A bitrate reduction of $1.1 \%$ on average compared to HEVC can be achieved, while further tests indicate that codebook adaptivity could substantially improve the performance.
\end{abstract}

Index Terms - HEVC, vector quantization, residue coding, intra prediction

\section{INTRODUCTION}

The High Efficiency Video Coding (HEVC) standard [1] developed by the Joint Collaborative Team on Video Coding has been ratified as an international Video Coding Standard in 2013. HEVC contains several elements improving the coding efficiency of intra prediction over the H.264/AVC standard. A wider range of coding block size is supported using a flexible quad-tree block partitioning structure, which allows the splitting of an image into Coding Units (CU) with sizes from $8 \times 8$ to $64 \times 64$. By introducing a larger number of prediction modes, the intra prediction in HEVC can more accurately model smooth regions as well as directional structures. Inside a CU, one or four Prediction Units (PU) are defined, each of which specifies a region with an individual intra prediction mode. Up to 35 intra prediction modes are available for a PU. A CU is further split into a quad-tree of Transform
Units (TU), on which transform, scalar quantization and entropy coding of residual signals are performed. The conventional intra prediction in HEVC is efficient at reducing the local spatial correlation in the pixel signals. However, the accuracy of intra prediction is limited in regions with complex textures or structures, so the residues in these regions have larger magnitudes.

In this paper, we focus on the remaining redundancy in the residual prediction domain. We propose a novel approach to encode the intra prediction residues based on a Mode Dependent Vector Quantization (MDVQ). A codebook is learned from training sequences and is optimized in a rate-distortion sense. It is shown that the codebook does not need to be adapted for each Quantization Parameter (QP), which is advantageous for the simplicity of the design. Furthermore, the method provides interesting bitrate savings, especially for low resolution sequences that are usually more difficult to compress.

The remainder of this paper is organized as follows. We introduce in the first part of Section 2 the method of residue coding using MDVQ in HEVC. In Section 2.2 we present the MDVQ codebook training procedure based on Rate Distortion Optimization (RDO). The QP-independent codebook construction is described in Section 2.3 and the new coding mode signalling is represented in Section 2.4. Experimental results are shown in Section 3. We finally conclude this paper and discuss the perspectives for future research in Section 4.

\section{RESIDUE CODING USING MODE DEPENDENT VECTOR QUANTIZATION IN HEVC}

The intra coding process of HEVC and H.264 constructs the intra predictor by extrapolating reference samples from previously reconstructed image blocks. In a block of samples predicted with planar mode or DC mode, the residual signals have a relatively homogeneous structure, whereas those derived from angular prediction modes tend to have directional 
structures. This residue characteristic has been previously investigated to improve coding efficiency. In [2], a directional transform and an adaptive coefficient scanning are used for coding the intra prediction residual. In our approach, a vector quantization codebook is learned with the aim of modelling the directional characteristics of the intra prediction residual signals.

Vector quantization [3] is an efficient data compression technique which exploits correlation in a vector of samples. Theoretically, better performance can always be obtained from coding vectors of source samples as a unit rather than individually. However, as the coding efficiency increases with the growth of the vector dimension, so does the size of required codebook and the complexity of searching for a best matching codeword. Since the codebook size is enormous for vectors of higher dimension, vector quantization is generally considered to be too complex to implement for practical use. We will demonstrate in the rest of this paper that, with our approach, good performance can be achieved for high dimensional vectors by using relatively small codebooks.

\subsection{MDVQ-based residue coding}

The proposed scheme of residue coding using vector quantization is shown in Fig. 1.

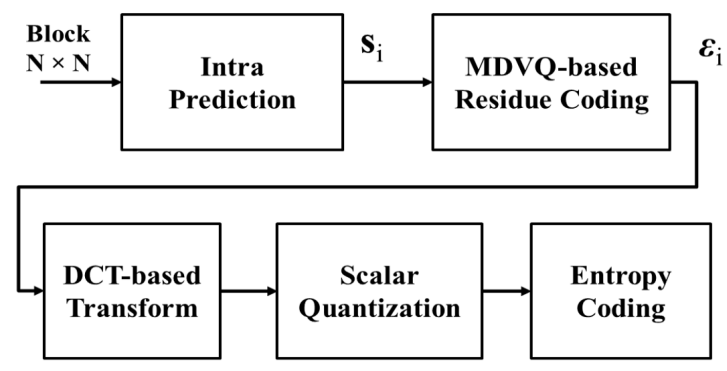

Fig. 1. The proposed approach of residue coding using mode dependent vector quantization

Firstly, an $N \times N$ image block is intra predicted by mode $i \in \mathcal{M}$ (the set of available modes) and the corresponding original residual signal $\mathbf{s}_{i}$ of the same dimension is computed. In the conventional HEVC coding chain, this is directly followed by transform coding, scalar quantization and entropy coding. Let us denote by $\mathbf{s}_{i}^{\prime}$ the reconstructed residual signal at the encoder, and by $\mathcal{R}\left(\mathbf{s}_{i}\right)$ the number of bits required to code the quantified transform coefficients. The best intra prediction mode $i^{*}$ is the one minimizing the Lagrangian cost function [4] [5]:

$$
i^{*}=\arg \min _{i \in \mathcal{M}} \mathcal{D}\left(\mathbf{s}_{i}, \mathbf{s}_{i}^{\prime}\right)+\lambda \cdot \mathcal{R}\left(\mathbf{s}_{i}\right)
$$

where $\lambda$ denotes the so-called Lagrange multiplier.

In our proposed scheme, a step of MDVQ-based residue coding is performed after the intra prediction. The $N \times N$ image block should be transformed into a $1 \times k$ dimensional vector $(k=N \times N)$ in order to be quantized by MDVQ using a pre-generated codebook. We will explain later in Section 2.2 how to generate this codebook. The index of the matching codeword will be then sent to the receiver and the final quantization error (the difference between the residual signal and the matching codeword) will be processed by a series of operations: DCT-based transform, scalar quantization and entropy coding.

Formally, let us denote by $\mathcal{C}_{i}$ the VQ codebook for intra prediction mode $i$, and by $\mathbf{v} \in \mathcal{C}_{i}$ a codeword in this codebook. Let $\mathcal{E}\left(\mathbf{s}_{i}, \mathbf{v}\right)$ be the quantization error of the input residual vector $\mathbf{s}_{i}$ quantized by vector $\mathbf{v}$ and $\mathcal{E}^{\prime}\left(\mathbf{s}_{i}, \mathbf{v}\right)$ be the reconstructed quantization error. The distortion measured on the prediction residue coded with MDVQ can be then expressed as:

$$
\begin{aligned}
\mathcal{D}\left(\mathbf{s}_{i}, \mathbf{v}\right) & =\left\|\mathbf{s}_{i}-\mathbf{v}-\mathcal{E}^{\prime}\left(\mathbf{s}_{i}, \mathbf{v}\right)\right\|^{2} \\
& =\left\|\mathcal{E}\left(\mathbf{s}_{i}, \mathbf{v}\right)-\mathcal{E}^{\prime}\left(\mathbf{s}_{i}, \mathbf{v}\right)\right\|^{2}
\end{aligned}
$$

Let $\mathcal{R}\left(\mathbf{s}_{i}, \mathbf{v}\right)$ represent the number of bits required for signaling the vector $\mathbf{v}$ in the codebook, plus the bits needed to code the quantization error. The best matching vector in a RD optimization sense for a prediction residue block is found by the following minimization:

$$
\mathbf{v}^{*}=\arg \min _{\mathbf{v} \in \mathcal{C}_{i}} \mathcal{D}\left(\mathbf{s}_{i}, \mathbf{v}\right)+\lambda \cdot \mathcal{R}\left(\mathbf{s}_{i}, \mathbf{v}\right)
$$

MDVQ-based residue coding will be used for a given residue block if the rate-distortion trade-off given by this mode satisfies:

$$
\mathcal{D}\left(\mathbf{s}_{i}, \mathbf{v}^{*}\right)+\lambda \cdot \mathcal{R}\left(\mathbf{s}_{i}, \mathbf{v}^{*}\right)<\mathcal{D}\left(\mathbf{s}_{i}, \mathbf{s}_{i}^{\prime}\right)+\lambda \cdot \mathcal{R}\left(\mathbf{s}_{i}\right)
$$

In other words, the MDVQ-based residue coding is performed only if the rate-distortion cost of coding an image block is reduced compared with the conventional HEVC coding.

\subsection{RD optimized codebook construction}

In the proposed method, the MDVQ codebooks are learned using training video sequences. The training set of residue vectors are obtained by extracting from the training video sequences the actual set of residual vectors that satisfie Eq. (4). As a consequence, the training set is not "polluted" by the residual vectors that would not be coded by MDVQ. In more details, an iterative approach is used in our codebook construction procedure as follows.

Iteration 0 :

- Step $A$ : The training video sequences are encoded with the conventional HEVC scheme (residue coding by MDVQ is not applied). Original residues $\mathbf{s}_{i}$ are gathered to form the training set for codebook construction. 
- Step B: The codebooks are constructed with $k$-means algorithm.

Iteration 1:

- Step $A$ : The training video sequences are encoded with residue coding by MDVQ, using the codebooks generated at the previous iteration. Original residues are extracted from TU blocks where residue coding by MDVQ is selected by RDO to build the training set for the next iteration. It should be noted that the MDVQ will only be selected for blocks where the condition described by Eq.(4) is fulfilled.

- Step $B$ : The codebooks are constructed with $k$-means algorithm.

Then the steps in Iteration 1 can be repeated several times. The process stops after a predefined number of iterations.

\subsection{QP independent codebook construction}

When a video sequence is coded with different Quantization Parameters (QP) in HEVC, the coding modes such as intra prediction mode or block partitioning can change. For instance, at higher QPs, less bits are used to describe the predictor, leading to a lower quality prediction, and an increase of the average energy of the residue. In order to take this into account, in our early experiments, four groups of MDVQ codebooks for QP values of 22, 27, 32 and 37 were learned separately. However, we have observed that the codebooks learned for different QP values were actually very similar. As a consequence, a generic MDVQ codebook has been learned by including in the training set of residue vectors obtained with the different QP values. The tests have confirmed that the same coding performance could be achieved using the QP independent codebook compared to the groups of codebooks learned separately for the different QP values. Therefore, a generalized QP-independent MDVQ codebook can be used. This property is very useful since it helps reducing storage requirements, and simplifies the design of the codec: it is not necessary to scale the vectors to match a certain ratedistortion compromise.

\subsection{New coding mode signalling}

In our experiments, MDVQ based residue coding is allowed only for $4 \times 4$ and $8 \times 8 \mathrm{TU}$ blocks. In reality, $4 \times 4 \mathrm{TU}$ blocks are much more frequently represented in $8 \times 8 \mathrm{CUs}$ than in $16 \times 16 \mathrm{CU}$ blocks, so we limit the residue coding by MDVQ on $4 \times 4$ TU blocks which reside inside a $8 \times 8 \mathrm{CU}$ block. For an $8 \times 8 \mathrm{CU}$ block which is split into four $4 \times 4$ TU blocks, we use three syntax elements and a tree structure for the signalization of residue coding by MDVQ. The syntax element $r e s_{-} v q_{-} c u_{-} f l a g$ indicates whether at least one of the four TU blocks inside the CU 8x8 block uses MDVQ. This flag is coded using one bit and an associated contextadaptive binary arithmetic coding (CABAC) context. When res_vq_cu_flag is set to 1 , for each $4 \times 4$ TU block a syntax element res_vq_tu_flag indicates whether MDVQ is used. This syntax element is also coded using one bit and an associated CABAC context. When MDVQ is used on a TU, a fixed-length syntax element res_vq_idx is transmitted, representing the index of the codeword in the codebook associated with the intra prediction mode and TU size. As the distribution of $8 \times 8$ TU blocks in different sizes of $\mathrm{CU}$ block is relatively uniform, only two syntax elements $r e s_{-} v q_{-} t u_{-} f l a g$ and $r e s_{-} v q_{-} i d x$ are signalling the use of MDVQ on $8 \times 8$ TU blocks. Except for the CU flag parsing step for $4 \times 4 \mathrm{TU}$ blocks, the reconstruction steps are shared between TU $4 \times 4$ blocks and TU $8 \times 8$ blocks.

\section{EXPERIMENTAL RESULTS}

The proposed approach has been used for residue coding of blocks when the TU block size is $4 \times 4$ and $8 \times 8$. For each of them, 35 codebooks corresponding to the 35 intra prediction modes have been derived using the codebook construction procedure described in section 2.2. Each codebook contains 256 codevectors, which represents a good compromise between storage, complexity and performance. However our tests indicate that further performance gains can be obtained with larger codebooks. The proposed method has been implemented in the reference software HM8.0 [6] of HEVC. The encoder is configured according to the JCT-VC common test conditions with the "all intra" profile. Sequences of six test classes with different resolutions specified in HEVC [7] were encoded. The experiments are performed under the midbitrate range of quantization parameters: $22,27,32$ and 37. The BD-Rate values are measured with the method in [8]. The performance is evaluated by comparing our method against HM8.0, where negative values indicate a bitrate saving.

\subsection{Training with test sequences}

In order to evaluate the potential of the learning method in the ideal case where the codebook is well suited to the input sequence, we have first learned the codebook using training vectors from the same sequence. Table 1 shows the performance of our method on class B sequences using this approach. In this ideal - but not realistic - case, one can observe that MDVQ can provide average bitrate savings of $4.9 \%$ on class B.

\begin{tabular}{|c|c|}
\hline sequence class & bit-rate saving (\%) \\
\hline Kimono1_1920x1080p & -0.25 \\
ParkScene_1920x1080p & -3.19 \\
Cactus_1920x1080p & -13.66 \\
BQTerrace_1920x1080p & -1.99 \\
BasketballDrive_1920x1080p & -5.16 \\
\hline average of class B & -4.95 \\
\hline
\end{tabular}

Table 1: Bitrate savings using sequence-dependent codebooks 


\subsection{Training with external sequences}

Table 2 shows the performance when the MDVQ codebooks are trained on sequences that are different from those used to measure the performance. This is the realistic use case. One can observe an average bitrate saving of $1.1 \%$. Interestingly, a larger gain is observed for low-resolution sequences which are usually more difficult to compress, this being attributed to the larger proportion of $4 \times 4$ and $8 \times 8 \mathrm{TU}$ blocks. The method also performs well on videoconference (class E) and screen content (class F) sequences. The higher performance shown in section 3.1 indicates that gains may be obtained with a realistic adaptive codebook approach.

\begin{tabular}{|c|c|}
\hline sequence class & bit-rate saving (\%) \\
\hline NebutaFestival & -0.09 \\
PeopleOnStreet & -1.62 \\
SteamLocomotiveTrain & 0.44 \\
Traffic & -1.51 \\
\hline average of class A & -0.7 \\
\hline BasketballDrive & 0.12 \\
BQTerrace & -1.33 \\
Cactus & -1.03 \\
Kimono & 0.02 \\
ParkScene & -1.44 \\
\hline average of class B & -0.5 \\
\hline BasketballDrill & -2.27 \\
BQMall & -1.34 \\
ParkScene & -0.95 \\
RaceHorse & -0.52 \\
\hline average of class C & -1.3 \\
\hline BasketballPass & -1.26 \\
BlowingBubbles & -1.24 \\
BQSquare & -0.98 \\
RaceHorse & -1.68 \\
\hline average of class D & -1.3 \\
\hline FourPeople & -1.61 \\
Johnny & -1.67 \\
KristenAndSara & -1.2 \\
\hline average of class E & -1.6 \\
\hline BasketballDrillText & -2.53 \\
ChinaSpeed & -1.29 \\
SlideEditing & -0.91 \\
SlideShow & -1.80 \\
\hline average of class F & -1.6 \\
\hline & \\
\hline
\end{tabular}

Table 2: Bitrate savings using sequence-independent codebooks

We show in Fig. 2 for the sequence RaceHorse of class $\mathrm{D}$, the blocks on which the MDVQ-based residue coding is performed.

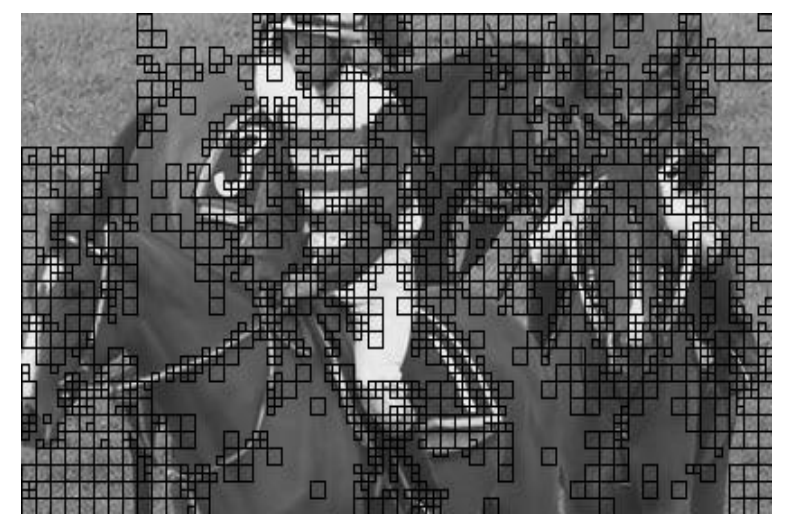

Fig. 2. Blocks on which MDVQ-based residue coding is performed

\subsection{Complexity aspects}

The proposed approach requires that codebooks are stored in the encoder and decoder. In general, vector quantization tends to impose a much larger complexity burden on the encoder (because of the search for the best codebook vector) than it does on the decoder (where a simple table look-up is needed). Therefore, it can be viewed as a tool that the encoder can optionally choose to use to further improve the compression, at the expense of some complexity, while the decoder can always easily support it. Furthermore, it is up to the encoder implementer to choose among the wide set of fast vector quantization methods (including tree-structured VQ) or other encoder-side optimization. For instance, to reduce the complexity, the use of MDVQ can be limited to the most frequent intra prediction modes.

\section{CONCLUSION}

We have presented a new approach for coding the intra prediction residues in an HEVC based encoder. This novel method can also be seen as a second-order prediction which aims to further remove remaining correlation in first-order intra prediction residues. The method is based on vector quantization with a codebook tailored for each intra prediction mode. Each codebook is hence learned considering a set of residual signals obtained when using a specific intra prediction mode. This training set of residual signals is further limited to the blocks for which the VQ will bring improved RD performance when compared to the reference HEVC encoding. Finally, these codebooks are QP independent, limiting the coder and decoder storage requirements.

\section{REFERENCES}

[1] W.J. Han G.J. Sullivan, J.R. Ohm and T. Wiegand, "Overview of the high efficiency video coding HEVC 
standard," in Transaction on Circuits and Systems for Video Technology. IEEE, December 2012, vol. 22, pp. 1646-1668.

[2] Y. Ye and M. Karczewicz, "Improved h.264 intra coding based on bi-directional intra prediction, directional transform, and adaptive coefficient scanning," in IEEE Conf. Image Process, October 2008, pp. 2116-2119.

[3] A. Gersho and R. M. Gray, "Vector quantization and signal compression," in Kluwer Academic Press. Springer, 1992, pp. 649-654.

[4] S.-W. Wu and A. Gersho, "Enhanced video compression with standardized bitstream syntax," in Proc. IEEE Int. Conf. Acoust., Syst., Signal Process. IEEE, April 1993, pp. 103-106.

[5] T. Wiegand, M. Lightstone, D. Mukherjee, T. G. Campbell, and S. K. Mitra, "Rate-distortion optimized mode selection for very low bit rate video coding and the emerging h.263 standard," in Transaction on Circuits and Systems for Video Technology. IEEE, April 1996, vol. 6, pp. 182-190.

[6] "Hevc test model (online)," http://hevc.hhi. fraunhofer.de/svn/svn_HEVCSoftware.

[7] F. Bossen, "Common test conditions and software reference configurations," in Joint Collaborative Team on Video Coding (JCT-VC) of ITU-T VCEG and ISO/IEC $M P E G$, February 2008.

[8] G. Bjøntegaard, "Calculation of average psnr differences between rd-curves," in ITU-T SG16 Q.6 Document. VCEG-M33, April 2008. 